\title{
EDITORIAL
}

\section{The Context of StichTrial: Viable or not viable Does it matter before revascularization?}

\author{
CHAUDHURY MESHKAT AHMED ${ }^{1}$, DR MD. ASHRAF UDDIN SULTAN ${ }^{2}$
}

University Heart Journal 2021; 17(2): 79-80

The concept of viability was unknown before 1980, until Rahintoola first describe the significant recovery of cardiac myocardial function after revascularization of coronary arteries. ${ }^{1}$ Eventually it discovered the chronically ischemic myocardium develop hibernation - a state of low metabolic function in the face of low oxyzen supply though stenosed coronary artery. The hibernating myocardium doesn't contribute functionally and in many a cases there is no thickening of ischemic myocardium during systole. This functional changes of myocardium often make it difficult to differentiate hibernating myocardium from dead myocardium.

The issue become more important in the context of infarct related myocardium, when the amount of viable myocardium dictates the outcome of revascularization in the infarct related artery, and the revasculirization is deffered in the absence of substantial viable myocardium.

Consequently many clinical and investigational measure specially the cardiac imaging modalities developed to detect myocardial viability and came into clinical use for deflection of viability before revascularization. The goal of viability assessment is to identifies the patients that would be benefited from revascularization and also to decide patient management in chronic heart failure as regards revascularization versus cardiac resynchronization therapy or left ventricular assist device.

But the utitility of widely practiced test for viability assessment before revascularization was challenged when the reports of stitch trial were published. Although Stitch trial as such was not designed to see the use of viability test. But the study analyzed a sub groups of study population, who underwent revascularization and have had viability test done prior to revascularization. During follow-up of 600 patients in the trial it was found that there was no difference in mortality between the groups of patients with or without myocardial viability. ${ }^{2}$ The findings of stich trial raised a big question about the use of viability test before revascularization and in many cardiac centers it went out of vogue.

The issue of assessing viability again has come into focus when in 2019 the New England Journal of Medicine published the follow up of the same patients from stich trial after 20 years of enrollement. At this point there was a trend towards better survival in the group of patients with viability than non viable group. ${ }^{3}$ The possible explanation for this findings could be due to the fact that it may take years for hibernating myocardium to function after revascularization and in some cases myocardial viability could not be delineated by single photon emission computerized tomography (SPECT) or by Dobutamine stress echocardiography (DSE). The Stich trial used only DSE and SPECT, so it is possible that some of patients from viable group were included in the non-viable group. The explanation is plausible, as with the newer imaging technique like Positron Emmission Tomography (PET) and magnetic Resonance Imaging (MRI) it is seen that these tests can detect myocardial viability in cases where SPECT or DSE failed to show the viability. ${ }^{4,5}$

Therefore, with the advent of imaging technique like PET and MRI for detection of viability, findings of stitch trial should no longer stand valid. Especially with MRI, where a segment thinner than $6 \mathrm{~mm}$ also show viability in some patients with significant improvement in left ventricular function after revascularization. ${ }^{5}$

So it seems prudent to use the viability test to determine the issues whether a patient should go for diagnostic angiogram or not. What should be the mode of revascularization if angiogram being done beforehand or what vessels should be targeted for revascularization. It is noteworthy that for detection of viable myocardium inference drawn from symptom or 12 lead ECG or resting or stress echocardiography may be sufficient in many cases. We should try to adapt this good practice of detecting myocardial viability before taking decision in selected patients.

${ }^{1}$ Professor Chaudhury Meshkat Ahmed, Professor, Department of Cardiology, Bangabandhu Sheikh Mujib Medical University, Dhaka

${ }^{2}$ Dr Md. Ashraf Uddin Sultan, Research Assistant, Department of Cardiology, Bangabandhu Sheikh Mujib Medical University, Dhaka 


\section{Reference:}

1. SH Rahimtoola. Coronary bypass surgery from chronic angina - 1981. A Perespective N Eng J Med 1982; 65(22); 225-41.

2. RO Bonow, G Manuer, KI Lae, TA Holly. Myocardial viability and survival in ischaemic left ventricular dysfunction, N Eng J Med 2011; 364: 1617-29

3. JA Panza, AM Ellis, HRA Khalide, TA Holly; Myocardial viability and long term out come in ischemic cardiomyopathy, N Enj Med 2019; 38; 739-48.
4. RSB Beanlands, G Nichol, F Huszti, D Humen, N Racine, M Freeman, Flurodeoxyglucose positron emission tomography imaging assisted management of patient with severe left ventricular dysfunction and suspected coronary disease,. controlled trial (PARR - 2) JACC 2007, Nov; 12; 30 (29) 2002-12.

5. DJ Shah, HW Kim, O James 1, Prevalence of regional myocardial thining and relationship with myocardial scarring in patients with coronary artery disease. JAMA 2013; 309 (9); 909-18. 